\title{
Iris Capture from Moving Subjects Using a Fluttering Shutter
}

\author{
Scott McCloskey, Wing Au, and Jan Jelinek \\ Honeywell ACS Labs, 1985 Douglas Drive North, Golden Valley, MN, 55422 \\ \{scott.mccloskey, wing.au, jan.jelinek\}@honeywell.com
}

\begin{abstract}
We address the problem of sharp iris image capture from moving subjects for the purpose of biometric identification. We utilize recent research from the field of computational photography, and capture images using the flutter shutter technique of [12]. Instead of capturing an image with traditional motion blur, we open and close the shutter several times during capture in order to effect invertible motion blur in the captured image. After applying automated blur estimation and de-blurring, we employ de-convolution to estimate a sharp image from the captured image. Through black-box testing with an existing iris matcher, we demonstrate the improved utility of these images for biometrics.
\end{abstract}

\section{INTRODUCTION}

Iris-based biometric identification [3] has great potential to enhance controlled access, law enforcement, and even surveillance of high-security areas. Like other applications that exploit visual information, however, iris-based biometrics is limited by the ability to acquire high-quality images in certain situations. Optical defocus due to a small depth of field (DOF) is one such problem, and recent work [5] has developed a system to mitigate its effects using image postprocessing. Another challenging situation is the acquisition of sharply-focused iris images from subjects undergoing lateral motion. Given a modest amount of light, the use of relatively long exposure durations results in motion blur, destroying crucial high-frequency information required for iris matching.

The field of Computational Photography has emerged in recent years, offering methods to mitigate both optical [7] and motion [12] blur. The underlying notion of Computational Photography is that the capture system (camera) and image processing methods should be jointly developed for a specific purpose. Though there is an extensive body of literature on estimating and removing motion blur from traditional images (e.g. [14], [15], [6], [2]), the elements of a traditional camera impose strict limitations on postprocessing.

The traditional open/close shutter is one such camera element. As we will discuss in Section IV, images of a moving object captured with a traditional shutter contain no information about the object at a number of spatial frequencies, and no image processing method can recover it from the image. Methods that attempt to de-blur the image will severely amplify sensor noise, hallucinate content, or both. Though it may be useful in improving subjective image quality, hallucinating image content is counter-productive for applications such as iris identification, and amplifying noise should certainly be avoided.
To avoid this loss of information during image capture, Raskar et al. have advocated the use of a fluttering shutter [12]. During exposure, the camera's shutter flutters between open and closed while exposure is accumulated on the sensor, producing an image with coded blur. Unlike traditional blur, which does not capture scene information at several spatial frequencies, coded blur conveys information about the subject at all frequencies. Given a suitably designed de-blurring method and an accurate estimate of the blur direction/extent, it is possible to recover a high quality image with information at all spatial frequencies and low levels of noise. Whereas images of a moving subject captured through a traditional shutter are often useless for iris recognition even after de-blurring, the de-blurred version of an image taken through a fluttering shutter will contain information necessary to determine the identity of the uncooperative subject. In this paper, we describe a preliminary study into the use of the flutter shutter technique for sharp iris image capture, based on a commercial, off the shelf (COTS) camera. We demonstrate, with synthetic and real images, that the use of the fluttering shutter allows us to capture recognizable images in situations where a traditional shutter cannot capture the necessary iris texture.

\section{ReView of Photographic TRAdeoffS}

Given the incremental progress in improving camera sensitivities (despite rapid increases in spatial resolution), the ability to capture high-quality images in challenging photographic settings depends on a number of well-known tradeoffs. In order to produce a minimum level of exposure from a dimly-lit scene, one or more of the camera parameters can be adjusted. The simplest, increasing the gain of the acquired signal, has the detrimental effect of increasing the noise component of the recorded image. A second option is to increase the scene's radiance using active illumination such as a flash. Because a flash's reflected illumination is essentially a point source illuminating a $3 \mathrm{D}$ region, its radiance falls off with the square of the distance, thus requiring very high illumination for distant subjects. The amount of light output by the flash is limited by a number of engineering considerations, but more fundamentally by eye safety, in that high-power flashes may cause physical damage to nearby people's retinas.

Given a fixed gain and scene radiance, then, the level of exposure is determined by the camera's shutter speed and aperture size. When motion blur is problematic, the most obvious solution is to reduce the exposure duration 


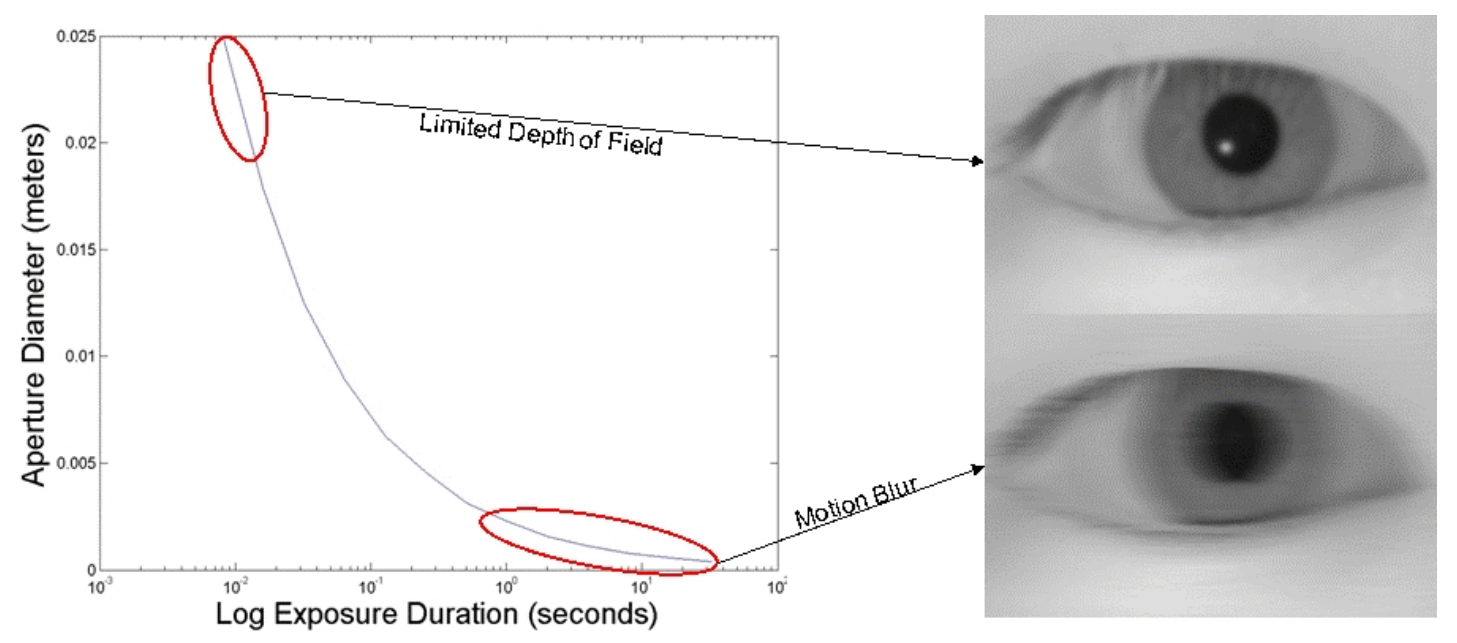

Fig. 1. The curve illustrates the pairs of aperture and exposure duration configurations that produce a fixed level of exposure. Use of wide apertures gives a limited depth of field, exacerbating problems of optical blur, whereas a long exposure duration results in motion blur.

and increase the size of the aperture. Figure 1 shows the combinations of exposure duration and aperture size that will result in the same level of exposure, which illustrates the fundamental photographic tradeoff. In order to acquire sufficient exposure of a scene with a low level of noise despite limited scene radiance (commonly found indoors) and without reducing the sampling resolution by binning pixels, one can use:

- a combination of long exposure and small aperture and suffer motion blur, or

- a combination of small exposure and large aperture and suffer reduced depth of field.

This tradeoff has existed since the advent of photography, and has already been exploited by engineers of optical biometrics systems.

Given the expected incremental nature of improvements to camera sensitivity, significantly expanding the operational capabilities of these systems requires a new way of capturing high-quality images of moving objects. The concept of a fluttering shutter does just that, capturing images that are useful over a wider range of exposure times. At acquisition, the fluttering shutter captures information about the subject that would be lost through a traditional open/closed shutter, as explained in Section IV. After a suitably-designed deblurring procedure, flutter shutter images have significantly richer texture than traditional shutter images, as we show in Section VI.

\section{Motion Sensitivity of Biometric Systems}

Whereas the previous section reviews some general photographic tradeoffs, it is also informative to understand the tolerance of iris matching biometric systems to motion blur. The current ANSI standard for iris images [1] specifies three levels (high, medium, and low) of image quality required for different applications. The least restrictive level (low image quality), intended for low security applications, specifies a resolution of 8.3 pixels per mm on the iris, and $60 \%$ contrast at a spatial frequency of 2 line pairs per millimeter. That spatial frequency corresponds to a line width of $0.25 \mathrm{~mm}$, meaning that subject motion of just $0.25 \mathrm{~mm}$ over the exposure duration would reduce the contrast to $0 \%$. In order to maintain the required $60 \%$ contrast, we approximate the upper bound of the lateral component of subject motion to be $0.025 \mathrm{~mm}$ over the exposure duration. Though the level of lighting is unlikely to permit it, the use of a $1 \mathrm{~ms}$ exposure duration means that the upper bound of subject velocity will be $0.025 \mathrm{~m} / \mathrm{s}$, or about $1 \mathrm{in} / \mathrm{s}$. Given that a person's walking speed is approximately $1.5 \mathrm{~m} / \mathrm{s}$, this is a significant limitation on (indoor) biometric acquisition.

Though interchange requirements may limit the degree of motion blur that can be accepted in biometric databases, it is also interesting to know how matching algorithms perform on motion-blurred images. Figure 2 shows ROC curves illustrating the performance degradation imposed by motion blur of various extents (details of this experiment are given in Section V). Though low-frequency iris texture allows for some robustness to motion, significant blur drastically reduces matching performance. In order to provide increased robustness to motion, then, images are typically post-processed. As we will demonstrate, post-processed images acquired using a fluttering shutter are significantly more robust to motion than post-processed images acquired using a traditional shutter. First, we review the theory behind the flutter shutter.

\section{Flutter Shutter: Theory And Implementation}

In order to properly motivate the use of a fluttering shutter, we briefly review the image quality implications of motion blur as seen through different shutters. A motionblurred image $I_{b}$ for linear, constant-velocity, lateral motion is traditionally modeled as the convolution of the (desired) sharp image $I_{s}$ with a 1D Point Spread Function (PSF) $B$,

$$
I_{b}=I_{s} \otimes B+\eta,
$$

where $\eta$ is Gaussian, zero-mean capture noise [12], and $\otimes$ represents convolution. Note that, when both the object and 


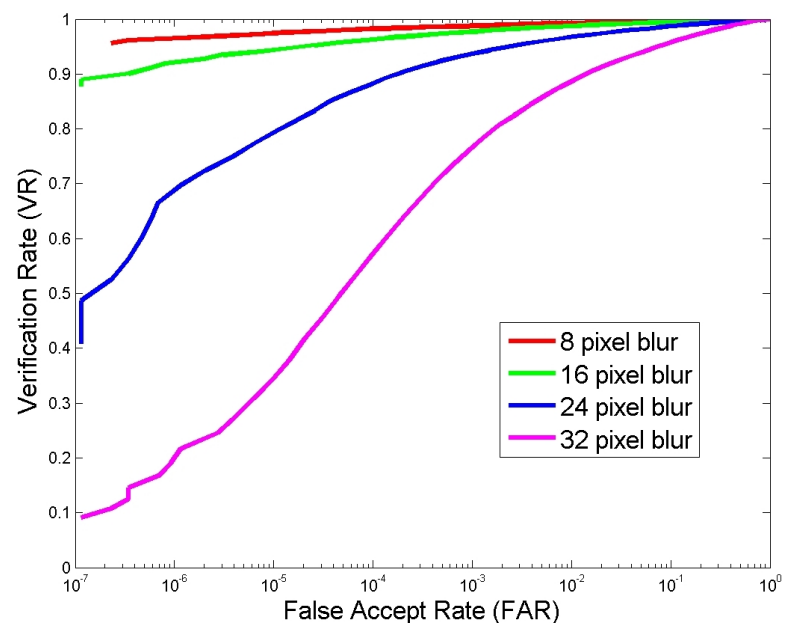

Fig. 2. ROC curves for synthetically blurred images from the ICE dataset [10], using a traditional shutter.

camera are stationary, $B=\delta$ (the Dirac delta function) and the image is unblurred.

In the frequency domain, at each frequency $k$,

$$
\hat{I}_{b}(k)=\hat{I}_{s}(k) * \hat{B}(k)+\hat{\eta}(k),
$$

where $\hat{I}_{b}, \hat{I}_{s}, \hat{B}$, and $\hat{\eta}$ are the Fourier transforms of $I_{b}, I_{s}, B$, and $\eta$, respectively. $\hat{B}$ is referred to as the Optical Transfer Function (OTF), and its absolute value as the Modulation Transfer Function (MTF).

The key contribution of the flutter shutter concept is that, in cases where $B \neq \delta$, the shutter's timing sequence should be chosen so that eq. 1 is invertible. Unfortunately, with a traditional open/closed shutter, $B$ is a rectangle function and inversion of eq. 1 is ill-conditioned (with respect to noise $\eta$ ) at several spatial frequencies. Fig. 3 (red curve) shows the MTF arising from a traditional shutter when the object moves over 26 pixels during exposure. The MTF shows that contrast is significantly muted at the middle and high spatial frequencies, and goes to zero at a number of spatial frequencies, at which the inversion of equation 1 is ill-posed. These spatial frequencies are lost when captured through a traditional shutter, and post-processing the image cannot recover that information.

Using the flutter shutter sequence from [12], on the other hand, gives rise to a filter $B$ whose MTF is shown as the blue curve in Fig. 3. $B$ is broadband, and preserves all frequencies at a nearly uniform level of contrast so that equation 1 may be inverted by de-convolution. Because the shutter opens and closes during image capture, the total capture time required to acquire the image is longer than the corresponding traditional shutter. In the experiments below, we compare traditional shutter images to flutter shutter images with the same exposure time, i.e. the total time that the shutter is open, though the flutter shutter images have a longer capture time (generally by a factor of 2).

Figure 4 shows an example flutter shutter image captured using our COTS camera, along with the de-blurred iris image produced by our method. In addition, the figure shows a de-

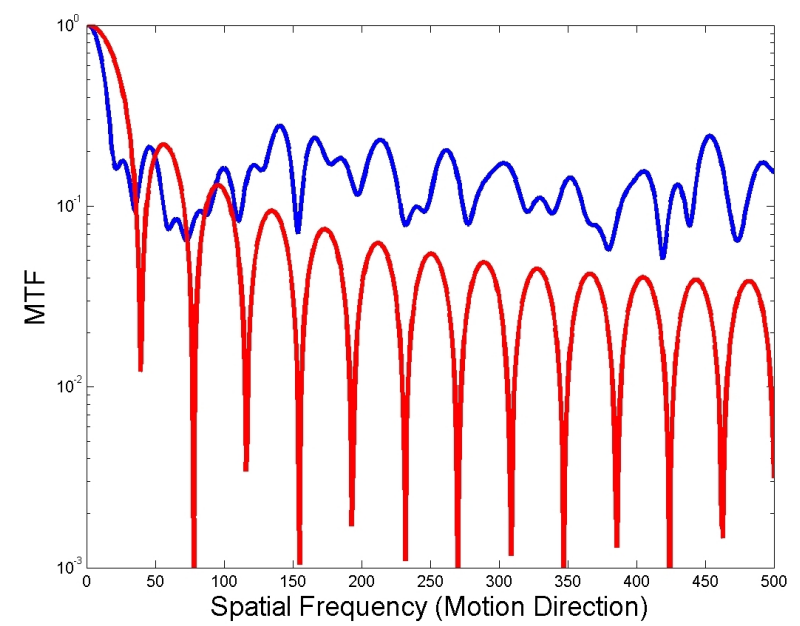

Fig. 3. The red curve shows the MTF of motion blur arising from a traditional shutter, which illustrates that several spatial frequencies are lost (i.e. the MTF goes to 0). The blue curve shows the MTF of motion blur arising from a flutter shutter, which has relatively uniform power at all spatial frequencies.

blurred image derived from a traditional shutter image, using Lucey-Richardson deconvolution [8], [13]. The flutter shutter image is recovered with significantly more detail in the high spatial frequencies, and has fewer artifacts due to lost spatial frequencies. Whereas neither the traditionally motion-blurred iris image nor its de-blurred result can be matched to a sharp image of the same iris, the de-blurred flutter shutter image can be matched. In order to quantify the improved utility of the flutter shutter images, we next describe experiments with real and simulated images.

\section{Synthetic IMAGE EXPERIMENTS}

In order to illustrate the improved robustness of flutter shutter imagery to motion, we have performed synthetic experiments using the NIST Iris Challenge Evaluation (ICE [10]) dataset. We simulate motion-blurred capture and deblurring, and compare those images to the sharp images of the dataset. We use eq. 1 to synthesize motion-blurred images for both the traditional and flutter shutters. In both cases, the noise $\eta$ has a standard deviation of 1 , and in both cases we use the ground truth blur kernel in the de-blurring step.

For the traditional shutter images, the blur PSF $B$ is a rectangle function of various widths: $8,16,24$, and 32 pixels, and we estimate an unblurred image using Lucey-Richardson deconvolution [8], [13]. For the flutter shutter images, the blur PSF $B$ is a flutter shutter PSF generated using the method of [9], with equivalent exposures to the $8,16,24$, and 32 pixel rectangle PSFs, and images are de-blurred using the de-convolution code provided in [12]. At a resolution of 8 pixels per $\mathrm{mm}$ on the iris, and for an exposure time of $2 \mathrm{~ms}$, these correspond to velocities of $0.5,1,1.5$ and 2 meters per second, respectively.

Comparisons of the iris images were fully automatic, and used Honeywell's POlar SEgmentation (POSE) template generation and matching algorithm. We emphasize that we treat these steps as a black box process, and that the 

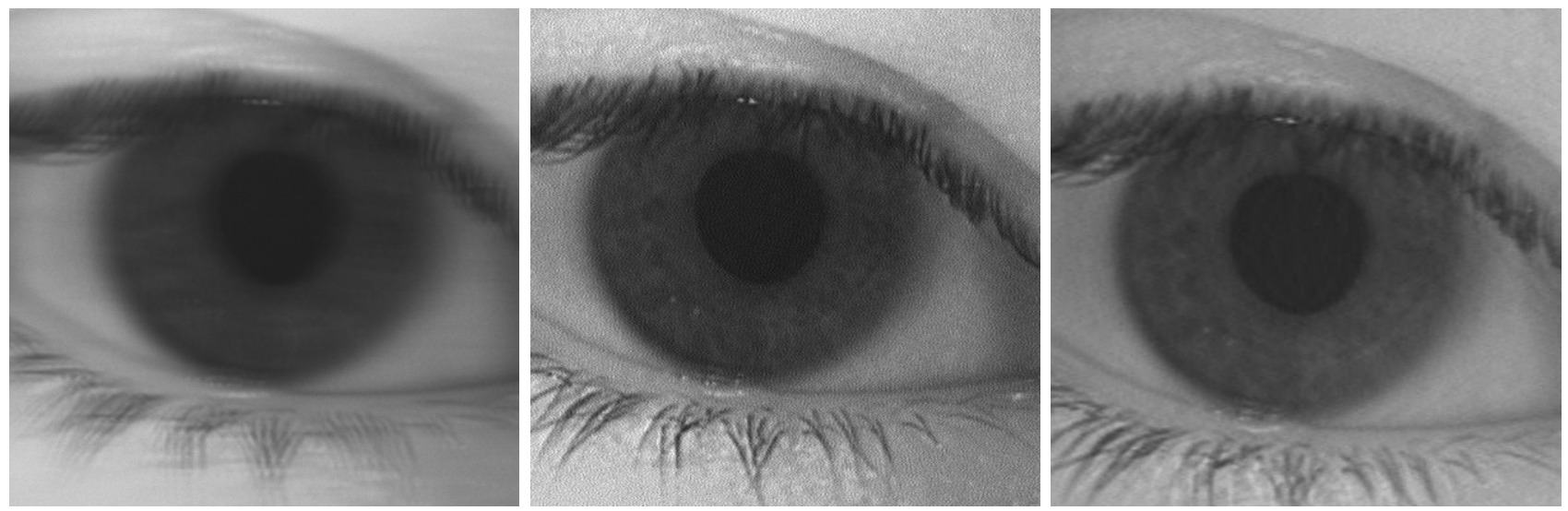

Fig. 4. (Left) Flutter shutter image. (Center) De-blurred flutter shutter image, which can be matched to an image of the same eye. (Right) De-blurred image acquired using a traditional shutter, which cannot be matched due to reconstruction artifacts (best noticed in the eyelashes).

algorithm is not optimized for de-blurred images. Though other template generation and matching algorithms will give different levels of baseline performance, we expect that the performance degradation imposed by motion blur and non-optimal de-blurring will be comparable for different algorithms, as they are related to quantifiable properties of the underlying images.

We have measured the Verification Rate (VR), False Rejection Rate (FRR) and the False Acceptance Rate (FAR) as performance metrics. For a given threshold Hamming distance $t$, two iris images are considered a computed match if the Hamming distance computed by POSE is less than or equal to $t$. VR, FRR and FAR are defined as:

$$
\begin{gathered}
F R R(t)=\frac{N_{r}(t)}{N_{s r}}, F A R(t)=\frac{N_{a}(t)}{N_{s a}}, \\
V R(t)=1-F R R(t), \text { where }
\end{gathered}
$$

- $N_{a}(t)$ is the number of iris matches that do not belong to the same subject as the test sample and the scores are lower than the threshold, $t$,

- $N_{r}(t)$ is the number of iris matches that belongs to the same subject as the test sample and the scores are greater than the threshold, $t$

- $N_{s r}$ is the total number of enrolled samples that belong to the test subject.

- $N_{s a}$ is the total number of enrolled samples that do not belong to the test subject.

Figure 5 shows the Receiver Operator Characteristic (ROC) curves for the synthetic images. The solid curves show the performance of the de-blurred traditional shutter images which, though they are greatly improved from the blurred images used in Figure 2, degrades sharply as the degree of blur increases. The dashed lines shown the performance of the de-blurred flutter shutter images, which show consistent performance despite increasing blur. These curves validate our claim that, when deblurred with the correct blur estimate, flutter shutter images are more useful than traditional shutter for iris matching.

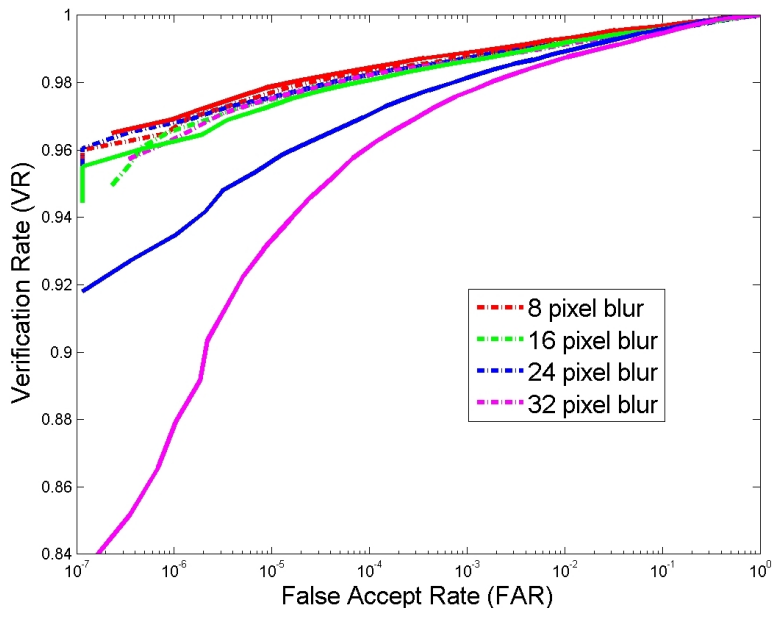

Fig. 5. ROC curves for synthetically de-blurred images from the ICE dataset, using a traditional shutter (solid lines) and a flutter shutter (dashed lines). Note the different scaling of the vertical axis, as compared to Fig. 2.

\section{REAL IMAGE EXPERIMENTS}

We have implemented a flutter shutter image capture system using the Point Grey Flea ${ }^{\circledR} 2$ camera, which supports multiple exposure capture [11]. The camera is tethered to a PC which controls the shutter via the serial port, and processes the captured image. As mentioned above, the captured image must be processed in order to estimate the underlying sharp image. The two-step processing involves:

1) Blur Estimation - Assuming that the underlying subject motion is linear with a constant velocity, we estimate the direction and extent of blur using a version of [4] that looks only at low spatial frequencies. The shutter sequence used to capture the image will, under different velocities, induce features in the blurred image's power spectrum, i.e. the local minima and maxima of the MTF, that are used to estimate the blur direction and extent.

2) De-blurring - Given the linear system of eq. 1, deblurring can be performed by de-convolving the filter $B$ from $I_{b}$. We use the code provided in [12]. 


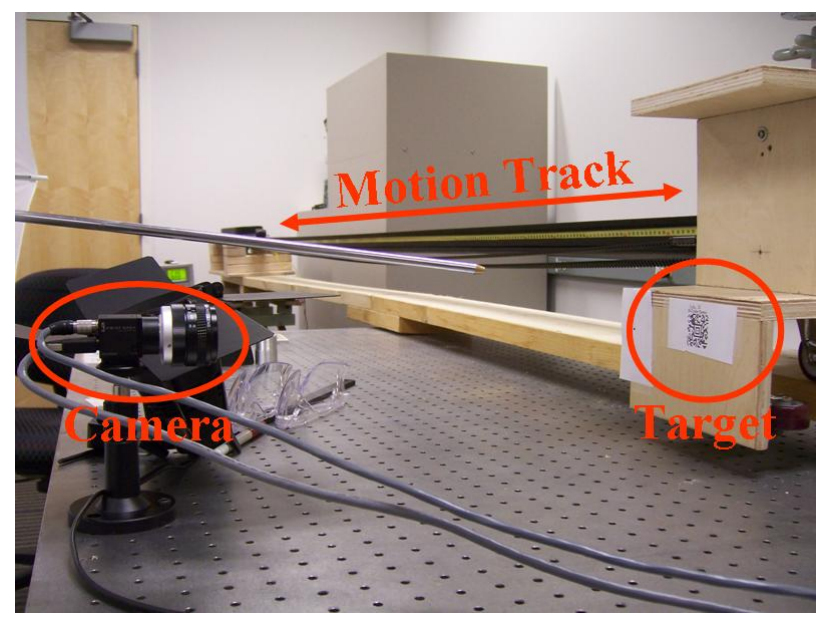

Fig. 6. Set up for stationary camera, moving subject experiments.

Our real image experiments were performed with a stationary camera capturing moving iris patterns, as shown in Figure 6. In this setup, the distance from the camera to the target is approximately $0.5 \mathrm{~m}$, and the target velocity can be manipulated via a voltage supply to the motor. The flutter shutter camera uses a lens with a $25 \mathrm{~mm}$ focal length and a variable aperture. The iris target is a 4-by-6 inch photographic print mounted on a moving track, and the camera is fixed to the optical bench. When the iris print is within the camera's field of view, the camera is triggered to acquire an image. The captured flutter shutter images were manually reviewed to make sure that the eye was visible and reasonably centered in the image.

\section{A. Real Image Dataset}

With respect to motion blur, the key nuisance factor is the distance that the iris travels during capture, i.e. the image's blur width. This distance is determined by two explicit settings of our test setup: the velocity of the target (controlled by a voltage supply to the motor) and the capture time of the image (which is determined by the camera settings to account for the amount of light). Hence the evaluation was performed against the test conditions under which the velocity and illumination are varied. Under all test conditions, the distance between the subject/iris print and the camera was fixed. As the light level is related to exposure time, when changing the level of illumination the exposure time was changed to compensate. Table VI-A lists the values of the parameters for the test conditions.

\begin{tabular}{|l|r|}
\hline Test Parameters & Values \\
\hline Velocity & $0.33,0.45,0.65,0.77 \mathrm{~m} / \mathrm{s}$ \\
Exposure time & $4 \mathrm{~ms}, 6 \mathrm{~ms}, 8 \mathrm{~ms}$ \\
\hline
\end{tabular}

We collected 600 ( 5 eyes $* 4$ velocities $* 3$ exposure times * 10 repetitions) flutter shutter images for our evaluation.

Having captured the flutter shutter images, we apply our automatic blur estimation and de-blurring to produce a processed image. We note that many of the de-blurred images had artifacts from one or more sources. One prominent source was edge effects in the de-blurring, which is particularly problematic when the iris was captured near the edge of the image. A second source was from erroneous blur estimates, where either the direction or extent of the blur differed significantly from the ground truth.

\section{B. Real Image Evaluation Protocol}

The de-blurred images were compared to a set of enrollment images, each of which was acquired from a stationary capture of the same image; for our preliminary experiments described here, the enrollment database contained only the five images from which the prints were generated. As in the synthetic experiments, comparisons of the iris images were fully automatic, and used Honeywell's POlar SEgmentation (POSE) algorithm. Each comparison yields a Hamming distance measurement between the two patterns, with values below a given threshold indicating a match.

Figure 7 (top) shows the ROC curve for selected images with reasonable image quality. In order to illustrate the fundamental performance of the flutter shutter technology, we have manually removed those images where the iris is distorted by edge effects in the de-blurring (due to the limited field of view of the lens), and those where the blur estimate is inaccurate, leaving approximately a third of the reconstructed images. From the ROC curve, we can see that the flutter shutter technique allows us to match $40 \%$ of the motionblurred images with a very small false accept rate of 0.001 , and increased proportions when higher false accept rates can be tolerated. An alternate view of the same results is shown in Figure 7 (bottom). It shows a histogram of Hamming distances, separated by those that correspond to true matches (blue curve) and those corresponding to mis-matches (red curve). As is clear from the figure, using a Hamming distance of 0.3 allows for a significant number of true matches without introducing any false positive matches.

Comparing the ROCs of Figures 5 and 7, it is clear that our real image system has not yet met the full potential of the technique. There are several causes for the differing results, most notably our use of estimated blur in the real image case. In addition, the model of eq. 1 assumes that motion is linear with constant velocity, that lighting is diffuse, and that the target has lambertian reflectance. Though most of these assumptions roughly hold in our experimental setup, there are deviations (as there would be a real-world application) that need to be addressed.

\section{CONCLusions \& Future Work}

Though Figure 7 (bottom) does not show a clear separation between the matches and non-matches (ideally, the curves would have no overlap), it illustrates the potential of the flutter shutter approach for iris acquisition. The ultimate use in an operational system requires further development to improve automated blur estimation. The results of our experiments with synthetic images illustrates the potential upper bound of performance, presuming that blur estimation can be perfected. Note that, even when automated blur estimation fails, the flutter shutter image still contains iris texture necessary to perform iris matching and a high-fidelity sharp image can be estimated using manual blur estimation. 

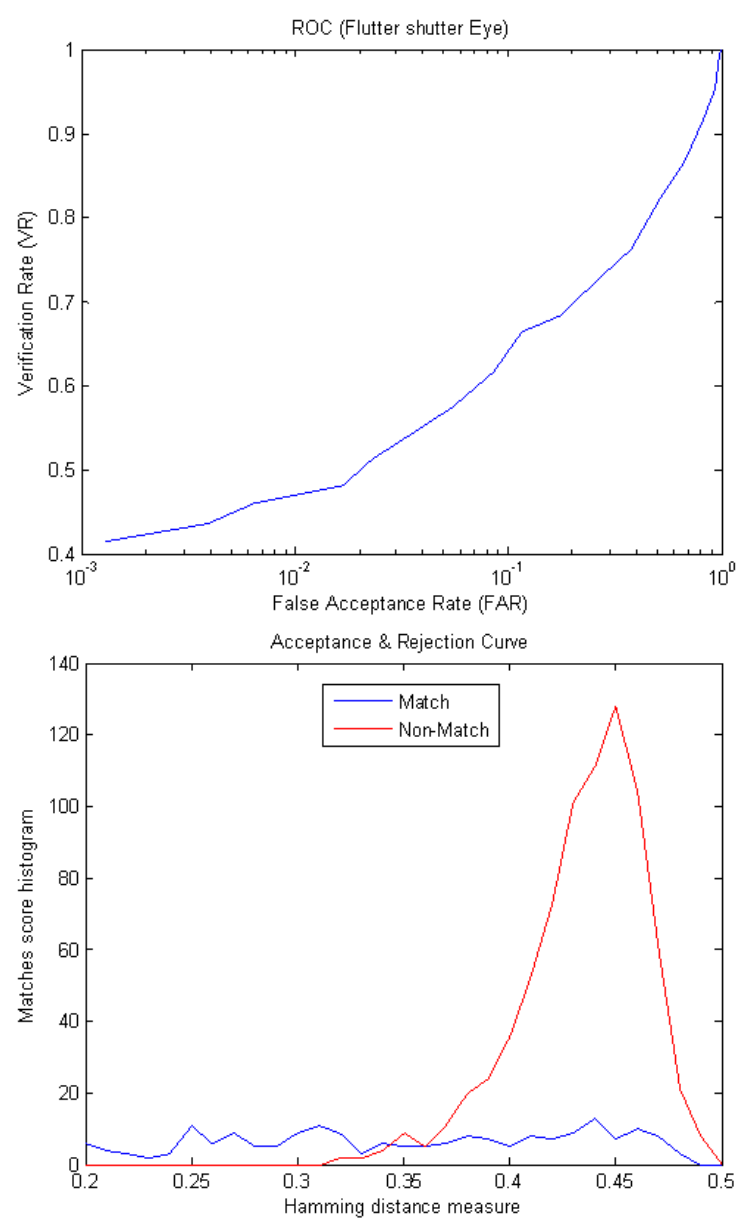

Fig. 7. (Top) ROC curve for selected images with accurate blur estimation. (Bottom) Histograms of Hamming distances for ground truth matches (blue curve) and ground truth mis-matches (red curve).

Though erroneous blur estimates are the greatest limitation of our current automated method, we note that there are several cases where qualitatively similar de-blurred images give surprisingly inconsistent matching results. Figure 8 shows two de-blurred images of the same iris print that, despite having qualitatively similar image quality, produce different matching results. Though this could easily be blamed on the specific iris matching technique that we have used, it is important to note that the images have qualitative differences due to slightly different PSFs. Though such differernces exist with images acquired with traditional photographic techniques, they are more acute when using the flutter shutter or other methods that require post-capture image processing. In addition to raising policy questions regarding the use of postprocessed imagery, this suggests that biometric matching techniques should take such processing into consideration. We have begun to analyze such methods.

In order to handle more general motion types than the constant velocity, lateral motion addressed in this work, we have also begun to investigate invertibility conditions for $2 \mathrm{D}$ motion blur corresponding to human gait.

\section{ACKNOWLEDGEMENTS}

This material is based upon work supported by the U.S. Army Biometrics Task Force under Contract No. W91CRB-
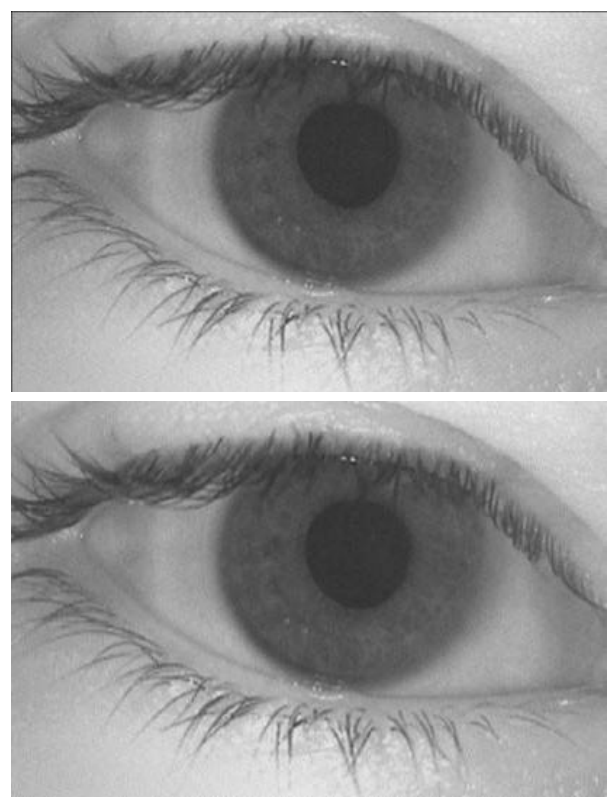

Fig. 8. Two qualitatively similar de-blurred flutter shutter images that produce different matching outcomes. The top image has a Hamming distance of 0.26 to the correct enrollment image (i.e. a true positive), while the bottom image has distance 0.37 (i.e. a false negative)

09-C-0013. Any opinions, findings and conclusions or recommendations expressed in this material are those of the author and do not necessarily reflect the views of the U.S. Army. Flea ${ }^{\circledR} 2$ is a trademark of Point Grey Research, Inc.

\section{REFERENCES}

[1] ANSI. Iris image interchange format ansi standard incits m1/03-0590.

[2] T. Cannon. Blind deconvolution of spatially invariant image blurs with phase. IEEE Transactions on Acoustic Speach Signal Processing, 24(1):58-63, February 1975.

[3] J. Daugman. High confidence visual recognition of persons by a test of statistical independence. IEEE Trans. Pattern Analysis and Machine Intelligence, 15(11):1148-1161, 1993.

[4] Y. Ding, S. McCloskey, and J. Yu. Analysis of motion blur with a flutter shutter camera for non-linear motion. In European Conf. on Computer Vision (ECCV), 2010.

[5] X. Huang, L. Ren, and R. Yang. Image deblurring for less intrusive iris capture. In IEEE Computer Vision and Pattern Recognition, pages 1558-1565, 2009.

[6] J. Jia. Single image motion deblurring using transparency. In IEEE Computer Vision and Pattern Recognition, pages 1-8, 2007.

[7] A. Levin, R. Fergus, F. Durand, and W. T. Freeman. Image and depth from a conventional camera with a coded aperture. ACM Transactions on Graphics (Proceedings of SIGGRAPH), 26(3):70, 2007.

[8] L. B. Lucy. An iterative technique for the rectification of observed distributions. Astron. J., 79:745+, June 1974.

[9] S. McCloskey. Velocity-dependent shutter sequences for motion deblurring. In ECCV, 2010.

[10] P. J. Phillips, W. T. Scruggs, A. J. OToole, P. J. Flynn, K. W. Bowyer, C. L. Schott, and M. Sharpe. FRVT 2006 and ICE 2006 large-scale results. In NISTIR 7408, March 2007.

[11] Point Grey Flea2 Camera Information. http://www.ptgrey.com/products/flea2/index.asp.

[12] R. Raskar, A. Agrawal, and J. Tumblin. Coded exposure photography: motion deblurring using fluttered shutter. ACM Transactions on Graphics (Proceedings of SIGGRAPH), 25(3):795-804, 2006.

[13] H. W. Richardson. Bayesian-based iterative method of image restoration. J. of the Optical Society of America, 62(1):55-59, January 1972.

[14] Q. Shan, J. Jia, and A. Agarwala. High-quality motion deblurring from a single image. ACM Transactions on Graphics (Proceedings of SIGGRAPH), pages 1-10, 2008.

[15] M. Sorel and J. Flusser. Space-variant restoration of images degraded by camera motion blur. IEEE Trans. Image Processing, 17(2):105116, February 2008. 